\title{
La storia della letteratura ladina delle Dolomiti e la letteratura ladina oggi
}

\author{
Rut Bernardi
}

Scrittrice

\begin{abstract}
L'autrice espone in modo succinto alcuni aspetti sulla letteratura ladina delle Dolomiti. Ne vengono esaminati concisamente sia le radici supposte, come anche i primi documenti reali scritti in lingua ladina. Viene anche fatto un piccolo confronto con la letteratura romancia dei Grigioni in Svizzera. Un ulteriore sguardo abbiamo dato agli scrittori ladini d'oggi e alla produzione letteraria con tutte le sue contraddizioni. Infine vengono proposti alcuni esempi scelti di opere letterarie ladine.
\end{abstract}

Parole chiave: Letteratura ladina, Dolomiti, confine, romancio.

\section{Abstract}

The author proposes a succinct exposition of several aspects of Dolomite Ladina literature: a concise examination of the supposed roots and the first real documents written in Ladina, and a brief comparison with the romantic literature of the Grigioni in Switzerland. He surveys the literary production of contemporary Ladini writers and its contradictions. Finally he offers some chosen examples of the Ladina literary oeuvre.

Key words: Ladin literature, Dolomites, frontier, romancio.

\section{Alcune ipotesi su lingue e letterature meno diffuse}

a) Alla denominazione usuale di «scrittori delle minoranze» noi preferiremo quella di «scrittori di lingue e letterature meno diffuse», perché la parola «minoranze» implica già qualche cosa di minore, mentre noi ci teniamo a dire che la gente e le opere (o le produzioni) di questi gruppi numericamente più piccoli, non hanno niente di minore, fuorché il numero di parlanti.

b) Un altro punto da chiarire è quello che riguarda il termine «minoranze». Ci sono i gruppi di una lingua maggioritaria, che a causa della storia e delle frontiere sono diventate «minoranze» di uno stato nazionale: il miglior esempio è la cosiddetta «minoranza tedesca» in Alto Adige. 
Sul territorio dello stato italiano sono naturalmente un gruppo etnico con una lingua meno diffusa. Però non sono una «minoranza linguistica» di per sè. Ci sono più di 70 milioni di persone parlanti tedesco, in diversi stati e naturalmente in tutte le varianti. E questa situazione la troviamo in Alto Adige anche dalla parte italiana. Gli italiani che vivono in Alto Adige sono numericamente in minoranza in rapporto alla provincia, ma hanno alle spalle un enorme serbatoio culturale, letterario, mediale, ecc.

Le vere minoranze sono le «lingue e letterature meno diffuse numericamente», come per esempio i ladini. La sola vera "minoranza» in Alto Adige è dunque per noi quella ladina.

c) Noi sosteniamo che gli scrittori, e naturalmente anche le scrittrici, di una lingua e letteratura meno diffusa sono quasi sempre in parte anche linguisti. Uno scrittore che scrive in una "grande» lingua, scrive, quasi sempre, come ha imparato a scrivere a scuola, mentre una scrittrice che scrive in una lingua meno diffusa deve sempre anche pensare a come scrivere, in quale ortografia, ecc.

d) Gli scrittori di lingue meno diffuse godono però anche di alcuni vantaggi: in queste letterature di "gente povera», come le chiama il filosofo e critico letterario del Canton Grigioni, Iso Camartin, ${ }^{1}$ si trovano, a volerle cercare, anche delle perle, come per esempio la libertà letteraria (una poesia dadaista in ladino è una novità, ma in tedesco è vecchia di 100 anni). Un «sistema linguistico grande» esprime anche una certa rigidezza, pensiamo al francese, ed è pure difficile da influenzare.

\section{Gli inizi della letteratura ladina, o meglio: i primi documenti in lingua ladina}

Prima di elencare i primi documenti e testi in ladino si deve fare un nome: Oswald von Wolkenstein, uno degli ultimi Minnesänger, poeta e cantore lirico dell'epoca cavalleresca. È un dato di fatto che una parte dei suoi beni erano situati laddove si parlava ancora ladino. Immaginiamoci Oswald in un'osteria di San Michele vicino a Castelrotto bisticciando con i contadini per la decima. È quasi impossibile immaginarselo in un'altra lingua che non sia quella ladina. $^{2} \mathrm{E}$ anche nel suo castello di Hauenstein a Siusi non possiamo imma-

1. Cfr. Iso Camartin, Nichts als Worte. Ein Plädoyer für Kleinsprachen, Zürich: Ex Libris, $1987^{2}$, p. 280.

2. Anche Wieland Schmied è certo che Oswald conoscesse il ladino: «Nicht ohne Bedeutung für seine dichterische Entwicklung ist gewesen, dass er schon als Kind mit dem rätoromanischen Idiom, das im Grödener Tal gesprochen wird und dem Provençalischen und Altfranzösischen näher ist als dem Italienischen, vertraut wurde.» (Per il suo sviluppo poetico non era secondario che avesse già da bambino contatti con l'idioma retoromancio, la lingua parlata in Val Gardena, che è più vicina al francoprovenzale e al francese antico che all' italiano.) Wieland Schmied, (Hg.), Oswald von Wolkenstein. Der mit dem einen Auge. Graz, Wien: Stiasny V., 1960, p. 10-11. 
ginarcelo in un'altra lingua che in ladino, quando s'infiltrava di nascosto nel camerino della sua amante, la moglie di Jäger.

Però, a parte gli scherzi, lui stesso elenca in una sua canzone, "Es fuegt sich, [...]», ${ }^{3}$ dieci lingue che ha parlato quando c'era bisogno, e l'ultima elencata la chiama: «român».

\section{Ma la lingua «român» era per Oswald von Wolkenstein il ladino?}

Beda Weber, il primo cronista e biografo delle poesie di Oswald parla della lingua «romanisch, römisch», ${ }^{4}$ come per dire «romanico, romano». Josef Schatz invece sostiene che è rumeno e ci rimanda alla poesia "Durch Barbarei, Arabia» dove si trova il verso "durch Romanei in Türggia». ${ }^{5}$ Questa "Romanei» è per Schatz la Romania. Nello stesso tempo troviamo che per Burghart Wachinger la lingua «român» è «ladinisch», ${ }^{6}$ ladino dunque, e il paese che Oswald ha attraversato si chiama «Byzanz». ${ }^{7}$ Anche Arthur Graf von Wolkenstein-Rodenegg definisce la «Romanei» come «das byzantinische Kaiserreich», 8 il regno bizantino.

Possiamo osservare che solo negli anni Sessanta si iniziò ad interpretare la lingua «român» come «ladino» oppure «romanisch = romanico», pur non sapendo esattamente quale lingua s'intendesse con «romanico». Anche Wieland Schmied interpreta la lingua «roman» come «romanisch»," "romanico» dunque. Tuttavia alcuni anni dopo si è già più prudenti nell'interpretare troppo sbrigativamente questa lingua e Heinrich Kuen scrive: «Zwar erscheint das Rätoromanische nicht unter den zehn Sprachen, der (sic!) er in diesem Lied aufzählt.» ${ }^{10}$ Ma subito dopo aggiunge: "Aber das ist kein Beweis, dass er diese Sprache nicht kannte, denn in einem anderen Lied gebraucht er zwei weitere Sprachen, die unter den zehn nicht genannt sind, nämlich Ungarisch und Flämisch.» ${ }^{11}$ Ed anche in una biografia di Oswald, pubblicata nel 1986, la lin-

3. Burghart WaChINGER, (Hg.). Oswald von Wolkenstein. Lieder. Mittelhochdeutsch / Neuhochdeutsch. Stuttgart: Reclam Jun., 1980², p. 38-39.

4. Beda WeBer, Die Gedichte Oswalds von Wolkenstein. Innsbruck 1847, p. 412.

5. Cfr. Josef SCHATZ, Sprache und Wortschatz der Gedichte Oswalds von Wolkenstein. (=Denkschriften, 69. Vol., 2. Abhandlung). Wien, Leipzig 1930, p. 4.

6. Burghart WaChInger, (Hg.). Oswald von Wolkenstein. Lieder. Mittelhochdeutsch / Neuhochdeutsch. Stuttgart: Reclam Jun., $1980^{2}$, p. 39.

7. Ibidem, p. 69.

8. Arthur Graf von Wolkenstein RodenEgG: «Oswald von Wolkenstein» in Schlernschriften 17, Innsbruck: Wagner, 1930, p. 6.

9. Wieland Schmied, (Hg.), Oswald von Wolkenstein. Der mit dem einen Auge, Graz, Wien: Stiasny V., 1960, p. 49.

10. Heinrich KUHN, «Rätoromanisches bei Oswald von Wolkenstein.» Ladinia 3, San Martin de Tor: Istitut Ladin "Micurà de Rü», 1979, p. 102. (Il retoromancio non si trova comunque tra le 10 lingue che elenca in questa canzone.)

11. Heinrich KuHN, ibidem (Ma cioè non è una prova che non conoscesse questa lingua, perchè in un'altra canzone usa due lingue ulteriori che non erano elencate tra le dieci, cioè l' ungherese e il fiammingo). 
gua «român» non viene più interpretata come «ladino»: «[...]; dass mit roman das Rätoromanische gemeint ist, wurde neuerdings wieder in Frage gestellt. Lüdtke nimmt an, es handele sich um das Okzitanische.» ${ }^{12}$ "Si deve anche prendere in considerazione che il numero dieci potrebbe solamente avere un valore simbolico", ${ }^{13}$ ci dice Ulrich Müller.

\section{E noi osiamo chiedere: la parola «schümpen» è ladina?}

La parola «schämpen» è segnata nel glossario di Josef Schatz con un punto interrogativo: «schämpen?» ${ }^{14}$ con il significato di: «ihr liesst das Knechtlein fahren.» ${ }^{15}$ Se Schatz non capiva questa parola, ci chiediamo se non possa essere una parola ladina, che esiste veramente. In ladino della Val Badia, in badiotto, «sciampé» ${ }^{16}$ vuol dire «andare via, spostarsi, scappare», ed anche in fassano «sciampèr» è «fuggire, scampare, scappare» ${ }^{17}$ (ortografia attuale).

\section{I primi documenti in lingua ladina:}

I primi documenti in lingua ladina sono già letteratura? Possiamo elencarli nel repertorio della nostra letteratura ladina? Queste sono le domande fondamentali che dobbiamo porci. Il critico letterario e saggista tedesco, Richard Alewyn, ci spiega che i testi anteriori al 1800 non possono essere considerati come letteratura. Non sono letteratura, ci dice, nel senso che diamo oggi alla parola letteratura, ma «letteratura di consumo», cioè una parte essenziale della vita di tutti i giorni del popolo analfabeta. ${ }^{18}$ Tra i primi testi in lingua ladina troviamo senźaltro opere di questo genere, ma ci teniamo a sottolineare che ce ne sono anche di quelli già «letterari».

1. 1568: Una poesia in ampezzano del 1568? Un certo Tiziano Vecellio dovrebbe aver scritto «24 verse par cadorin par sta Marieta». Ernesto Majoni nella «Usc di Ladins», 26.9.1992, p. 13.

12. Karen BaAsCH; Helmuth Nürnberger, Oswald von Wolkenstein, Reinbek bei Hamburg: Rowohlt, 1986, p. 131, («[...] che con roman è inteso il retoromancio, è ultimamente di nuovo stato messo in dubbio. Lüdtke pensa che si tratti dell' occitano. (Lüdtke, 305ff).»

13. Cfr. Ulrich MÜLler,: “Dichtung” und "Wirklichkeit” bei OvW, aufgezeigt im Vergleich mit Altersliedern von Walther von der Vogelweide und Hans Sachs" Literaturwissenschaftliches Jahrbuch. NF 19, 1978, p. 133-156.

14. Cfr. Josef SCHATZ, op. cit., p. 81, 43.

15. Ibidem, p. 94. ("voi avete lasciato scappare il servo»)

16. Antone PIZZININI, Parores ladines. Vokabulare badiot - tudësk. Ergänzt und überarbeitet von Guntram Plangg, Innsbruck 1966. (shampé, -a- [ 1a ] ausweichen, aus dem Weg gehen; s'enn - fliehen)

17. DILF: Dizionario italiano - ladino fassano. Vich / Vigo di Fassa: Ist. Cult. Lad.-Spell, 1999, p. 543.

18. Cfr. Richard Alewyn, Probleme und Gestalten. Essays. Frankfurt: Suhrkamp, 19886, p. 307. 
2. 1631; 1632: I "proclami» (cridati) dalla chiesa per il popolo in lingua ladina: $1631^{19}$ in fassano ${ }^{20}$ (fascian) e nel 1632 in livinallonghese (fodom).

3. 1703: Un "proclama" di 250 parole in ladino della Val Badia, il badiotto (badiot), per la chiamata dei soldati alle armi. ${ }^{21}$ (Die aus dem Gadertal stammende «ladinische Übersetzung einer Proklamation des Bischofs Kaspar Ignaz von König (1702-1747) [...] an die Thurner (Unteres Gadertal) Untertanen» ist «für den Rätoromanisten ein sehr aufschlussreiches und wichtiges Literaturdenkmal, das [...] den frühesten zusammenhängenden Text des Dolomitenladinischen darstellt.» ${ }^{22}$

4. 1760: Una lista di parole in ladino badiotto di Simone Pietro Bartolomei, avvocato di Pergine: Catalogus multorum verborum quinque dialectum, quibus montani Perginenses, Roncegnenses, Lavaronenses, Septem Pagenses et Abbatienses utuntur.

5. 1800: J. Insam, avvocato a Gudon: "Gramatica dl gherdëina». Irreperibile.

6. 1807: Matie Ploner: 6 aneddoti popolari corti e 150 parole gardenesi. («La vedla muta», «L vedl mut»). ${ }^{23}$

7. 1813: Johann Peter Runcaudie: «La Stacions o' la via della S. Crousch» (Le stazioni o la via crucis) in gardenese.

8. 1836: Una traduzione del catechismo con i dieci comandamenti in badiotto.

9. 1844: Joani Gregorio Domenego Caisar (1821-1867): «Satira bela longa e piena de pear» (Satira bella lunga e piena di pepe) in ampezzano.

10. 1848: Cyprian Pescosta: «Badiotisches Schützenlied» (Canzone degli Schützen in badiotto).

11. 1856: Don Giuseppe Brunel (1826-1892): una poesia di 30 quartine per una messa novella. Inoltre ha scritto testi di teatro e racconti popolari.

12. 1865: Jakob Pitscheider, Karl Maneschg und Putzer: un catechismo ampio, tradotto in badiotto.

13. 1879: Matteo Declara,: "Storia d' S. Genofefa» (La storia della Santa Genofefa). "Prum liber lading» (Primo libro ladino) dell'anno 1879.

14. (1862-1888): Angelo Trebo, 24 poesie e alcune opere teatrali.

19. Frumenzio GhetTA; G.A PlangG, «Un proclama Ladino del 1631» Mondo Ladino XI, 1987 3-4, (Vich / Vigo di Fassa: Ist. Cult. Ladin), p. 281-293. (Il testo più antico in ladino dolomitico venne trovato nell' "archivio dei conti Wolchenstein-Trostburg», l'archivio statale di Trento. È un proclama dell'anno 1631, una specie di comunicazione alla popolazione fassana. Questi proclami vennero "cridati» sulle piazze dei paesi nelle valli ladine.)

20. Fabio CHIOCCHETTI, "Appunti per una storia della letteratura ladina dolomitica» Mondo Ladino XXIV, 2000 (Vich / Vigo di Fassa: Ist. Cult. Ladin), p. 15. ("[...] in occasione delle fiere di San Giovanni, nei quali si parla di "vidis, gialines, capuns, ponjins, smalz, us”, cioè "vitelli, galline, capponi, burro, uova e merci consimili" [...]»).

21. G.A. PlANGG, "Gadertaler Ladinisch um 1700" Romanica Aenipontana X, 1976 (Innsbruck, Inst. f. Rom. Phil.), p. 129-146.

22. Ibidem, p. 129. (Questa traduzione in ladino proveniente dalla Val Badia è un proclama del vescovo Kaspar Ignaz von König (1702-1747) ai sudditi della Bassa Val Badia ed è per il retoromanista un monumento letterario di grande importanza in quanto rappresenta il più antico testo continuo scritto in ladino dolomitico.)

23. Josef STEINER, Die Grödner (= Der Sammler für Geschichte und Statistik von Tirol). II, Vol. 1. Innsbruck, p. 1-52. 
15. 1864: J. Antone Vian: «Gröden, der Grödner und seine Sprache». (Gardena, il gardenese e la sua lingua). La prima grammatica ladina in lingua tedesca con testi di prosa in ladino.

16. (1864-1930): Jepele Frontull: opere teatrali e musicali.

17. 1865: Joh. Aug. Perathoner: «Nsenyamént per la zoventù» (Insegnamento per la gioventù). 107 versi. Un'opera teatrale del XVI secolo tratta dal francese di Merch Tòne Murèt (Marc Antoine Muret).

18. 1878: Diversi testi di Giuani Batista Alton, verso la fine dell’ Ottocento. («L gran Bracun», «La fana de Val de Mesdì»)

19. 1879: Theodor Gartner, alla fine del 1800: «Die Gredner Mundart» con alcuni testi letterari in ladino.

20. 1900: Rosalia Riffeser Comploj: «La pasion y mort de nosch Senieur GesuCristo", 257 versi. Notazione di un testo tramandato a voce.

21. 1905: Hugo de Rossi in fassano: «Ko ke la e stada ke son rua sul Ball dei Dolomitenladiner» ${ }^{24}$ (Come è successo che sono arrivato sul ballo dei Ladini delle Dolomiti). ${ }^{25}$

22. 1905 / 1908: Wilhelm Moroder: 2 giornali: «L'Amik di Ladins» (3). Innsbruck, 1905; «L'Ladin» (2). Bressanone, 1908.

23. (1911-1915): I «Calëndri de Gherdëina» (Calandari della Val Gardena). Pubblicati da: A. Lardschneider, J. Runggaldier da Passua, F. Moroder: «Doi vödli Gherdëines», «Doi vödli jagheri». Con molti testi in prosa e poesie. P. es.: «La vedla muta», «L vedl mut».

24. 1913: Enghel da Plazola (Demetz) e Giuani da Pertèut (Perathoner): «Pitla Storia Bibia» (La piccola storia della Bibbia).

25. 1921: Leo Runggaldier da Furdenan «Stories i Cianties per kei de Gerdeina» (Storie e canzoni per la gente della Val Gardena).

26. 1922: Hugo de Rossi: «L Saut de Jókele te la libertà», na kontìa braka. (Il salto di Jókele nella libertà, una storia in fassano brak). ${ }^{26}$

\section{Alla ricerca delle vere radici della letteratura ladina}

Questi sono i risultati scientifici e i documenti trovati ${ }^{27}$ e secondo questi dati, siamo d'accordo con Fabio Chiocchetti che «la nascita della letteratura ladina nelle Dolomiti ci appare innanzi tutto come un fenomeno piuttosto tardivo rispetto alle altre lingue minoritarie, e alle altre lingue romanze in genere». ${ }^{28}$

24. Cfr.: Fabio ChiocchetTi, «† Hugo de Rossi (1875-1936) Ko ke la é stada ke son ruà sul bal dei Dolomitenladiner. Introduzione» Mondo Ladino VI,1-2, 1982 (Vich / Vigo di Fassa: Ist. Cult. Ladin), p. 121-191.

25. Ibidem, p. 127: (una trascrizione del 1912 di un racconto umoristico composto nel 1905 in occasione di una festa organizzata dai ladini a Innsbruck).

26. Fabio CHIOCCHETTI, op. cit. p. 127.

27. L'autrice di questo articolo non può garantire di aver trovato ed elencato tutti i primi documenti esistenti scritti in ladino dolomitico.

28. Fabio ChIOcCHeTtI, "Appunti per una storia della letteratura ladina dolomitica» Mondo Ladino XXIV 2000 (Vich / Vigo di Fassa: Ist. Cult. Ladin), p. 12. 
Però secondo noi questi documenti non sono talmente importanti da poter affermare, per esempio, e neppure da poter immaginare, che Oswald sapesse parlare ladino. Secondo noi, nella letteratura ladina, bisogna prendere in considerazione altre caratteristiche ben più importanti: l'immaginazione e la fantasia, fondamentali per avvicinarsi e per capire la letteratura ladina. ${ }^{29}$ Solo considerando questo aspetto filosofico ${ }^{30}$ e meno scientifico si possono trovare le vere radici della letteratura ladina nelle leggende, le «lijëndes», cioè nella letteratura orale.

La tradizione del tramandare a voce di generazione in generazione le leggende delle Dolomiti, e cioè la nostra letteratura ladina, richiede una valutazione diversa da quella scientifica. Richiede un approccio più aperto, simile a quello della scienza letteraria popolare.

Per questa ragione osiamo affermare che la letteratura ladina non inizia con una poesia o un proclama, anche se sono questi indubbiamente i primi documenti scritti in questa lingua. E considerando anche le affermazioni di Richard Alewyn, citato sopra, riguardo alla sua definizione di che cosa è letteratura, dichiariamo che la letteratura ladina inizia con le leggende delle Dolomiti, con «la lijëndes», scritte in tedesco, ma molto più letterarie, nel senso artistico, di tutti i primi documenti scritti in ladino. Ricordiamo che la prima codificazione piuttosto libera delle leggende delle Dolomiti è stata fatta in tedesco all'inizio del 1900 dal giornalista Karl Felix Wolff nella tradizione romantica tardiva dei fratelli Grimm. Ma anche se Wolff ha raccolto le leggende ladine in lingua tedesca, i ladini gli sono molto grati, perchè è stato lui a salvarle dall'oblio.

La trama originale delle leggende non la conosceremo mai, ci dice Ulrike Kindl. ${ }^{31}$ E come Kindl, osiamo affermare che la conoscenza esatta del contenuto delle leggende non è rilevante, solamente la nostra fantasia è importante, cioè è la nostra capacità di immaginazione ad essere rilevante. Ciò che è determinante è come riusciamo ad assumere gli eventi leggendari, con tutte le loro varianti, nella nostra immaginazione, seguendo il principio del «Mondo come immaginazione» descritto da Kant e Schopenhauer. ${ }^{32}$

Un esempio prezioso in questo senso ce l'ha regalato la scrittrice altoatesina Anita Pichler ${ }^{33}$ nel suo libro «Die Frauen aus Fanis», ${ }^{34}$ in cui propone una sua interpretazione delle figure femminili nel ciclo delle leggende di Fanes.

29. Kindl Ulrike in una relazione tenuta il 7 marzo del 1994 nel Circolo Culturale di Ortisei in Val Gardena.

30. Arthur Schopenhauer, Die Welt als Wille und Vorstellung. Vol. I, vol. II, Zürich: Haffmans V., 1988.

31. Professoressa di letteratura popolare all'Università di Venezia, ma oriunda di Lana in Alto Adige.

32. Kindl Ulrike in una relazione tenuta il 7 marzo del 1994 nel Circolo Culturale di Ortisei in Val Gardena.

33. Anita Pichler (1948-1997).

34. Anita Pichler; Markus VallazZa, Die Frauen aus Fanis. (Le donne di Fanes), Insbruck: Haymon V., 1992. 


\section{Un confronto con i romanci del Canton Grigioni}

I primi documenti scritti in ladino e romancio su territorio grigionese, sono dell'XI-XII secolo, qualora non si prenda in considerazione la nota canzone «La Canzun da Sontga Margriata» (La canzone della Santa Margherita), perla della letteratura romancia, tramandata a voce di generazione in generazione. Questi primi documenti sono annotazioni interlineari, trovate nel convento di Einsiedeln: ${ }^{35}$ tra le righe scritte in latino, un monaco ha inserito alcune note personali in romancio per una miglior comprensione del testo.

Più avanti abbiamo Gian Travers di Zuoz (Engiadina Alta) con la sua epopea in versi "Chianzun dalla guerra dagl Chiaste da Müs» ${ }^{36}$ (Canzone della guerra al castello di Müs) del 1527. Ma i primi libri stampati erano traduzioni della Bibbia in «ladin puter», il ladino della Engiadina Alta nel 1562 di Giachem Bifrun. ${ }^{37}$ Questo fu solamente l'inizio di una serie di testi religiosi in ladino e in romancio, nati in seguito ai conflitti tra la Engiadina protestante e la Surselva cattolica. Nelle zone ladine delle Dolomiti mancava completamente questo impulso.

C'è un'ulteriore e fatale lacuna nella letteratura ladina delle Dolomiti. Nel Canton Grigioni esiste la «Rätoromanische Chrestomatie» ${ }^{38}$ compilata da Caspar Decurtins di Trun della Surselva (1855-1916). Quest'opera monumentale, che secondo P. Maurus Carnot è un «monumento retico», comprende tutti i migliori testi grigionesi scritti in romancio, «ina raccolta dil meglier» (una raccolta delle migliori opere), come Decurtins la definisce, in 13 volumi per complessive 7.260 pagine e con una introduzione di 120 pagine con commenti. Il primo volume è uscito nel 1896 e l'ultimo nel 1919. Decurtins chiarisce che il maggior valore della «Chrestomatie» romancia è senza dubbio la raccolta delle tradizioni orali di genere popolare, che in questo modo si sono potute salvare. ${ }^{39}$ Anche una raccolta delle tradizioni orali scritte in ladino manca completamente nelle Dolomiti.

\section{Inizio della letteratura ladina classica}

Per datare l'inizio della letteratura ladina classica oso fare un'ipotesi, affermando che si apre con i testi di Leo Runggaldier da Furdenan nel 1921 e propongo una serie di autori che hanno inciso profondamente nel panorama culturale e letterario ladino. ${ }^{40}$

35. Cfr. Reto R.Bezzola, Litteratura dals Rumauntschs e Ladins, Cuira: Lia Rumauntscha, 1979, p. 126-127.

36. Ibidem, cfr. p. $152-153$

37. Ibidem, cfr. p. 190.

38. Caspar DeCURTINS, Rätoromanische Chrestomathie, Erlangen, 1896-1919. 13 Vol.

39. Cfr. Alexi DeCurTins, "Entgins patratgs davart la Crestomazia e siu editur», Viarva romontscha II. (=Romanica Raetica 9) 1993 (Cuira: Societad Retorumantscha), p. 106111.

40. Cfr. Walter Belard, Antologia della lirica ladina dolomitica, Roma: Bonacci, 1985. 
Gherdëina / Val Gardena: Adele Moroder de Lenèrt (1887-1966), Max Tosi (1914-1988), Frida Piazza (1922), Josef Kostner (1933).

Badia cun Mareo / Val Badia con Marebbe: Angelo Trebo (1862-1888), Angel Morlang (1918), Pio Baldissera (1929), Lois Ellecosta (1938), Felix Dapoz (1939), Iaco Ploner (1944), Giuvani Pescollderungg (1937)

Fascia / Val di Fassa: Valentino Dell'Antonio (1909-1981), Luciano Jellici (1928), Simone Sommariva (1927), Veronika Zanoner Piccoljori (1922)

Fodom / Livinallongo: Franco Deltedesco (1942), Sergio Masarei (1943) Ampez / Cortina d'Ampezzo: Marco Dibona (1962)

Nell'ambito della definizione «letteratura classica» credo possano essere citati questi autori ed i loro testi, sebbene tra essi ce ne siano alcuni al limite della modernità, come per esempio i testi «curc ma sëc» (corti ma forti / intensi) di Josef Kostner, oppure le rime atemporali ed estremamente musicali di Frida Piazza.

\section{La letteratura ladina oggi}

\subsection{Scrittrici e scrittori}

Scrittrici e scrittori, ma sarebbe meglio chiamarli «scriventi», ${ }^{41}$ sono il più delle volte maestre e maestri oppure artisti del cosiddetto ceto intellettuale dei paesi ladini, anche se è molto difficile definire tale ceto nelle nostre valli appartate. Non esistono scrittori che scrivono in lingua ladina e che esercitano questo lavoro come professione. Non ancora. E non solo nelle vallate ladine delle Dolomiti, ma anche nel Canton Grigioni, dove la tradizione letteraria è assai più lunga, non ci sono scrittori professionisti. ${ }^{42}$

Ma chi sono queste persone che scrivono e pubblicano, malgrado tutte le difficoltà, ${ }^{43}$ poesie, romanzi, oppure testi teatrali in lingua ladina? Ecco alcuni nomi:

\section{Gherdëina / Val Gardena:}

Bernardi, Rut: Rumantsch grischun y ladin dolomitan - ntraunida lenguistica. Turic: edizion «L Brunsin», 1991. (Dramma)

Bernardi, Rut: Stories de Giuani da Bula. Stories de Gion Barlac de Theo Candinas, Tluses: U.L.G., 1993. (Prosa-traduzione dal sursilvano)

Bernardi, Rut: Lëtres te n fol, Cuntì: Tluses, Edizione propria, 1996. (Romanzo-racconto)

41. Usiamo il termine «scriventi» per persone che scrivono letteratura ladina per evitare il termine "scrittori" che non ci sembra corretto, perchè potrebbe indicare un mestiere.

42. Solamente Flurin Spescha di Domat nel Canton Grigioni (1958-2000) ho sentito rispondere alla domanda sul suo mestiere: "Io sono scrittore.» Spescha, però, non scriveva unicamente in lingua romancia, scriveva anche in tedesco.

43. Fino ad oggi non esiste una casa editrice che abbia pubblicato letteratura ladina pura. Per la prima volta, nella primavera del 2003, la casa editrice «Studienverlag-Skarabäus» di Innbruck pubblicherà «4 corone di sonetti» (60 sonetti) in lingua ladina con traduzione tedesca più un $\mathrm{CD}$ audio di Rut Bernardi. 
Bernardi, Rut, Nëus jon cun la ferata. 6 stories crimineles, Tluses: Edizione propria, 2002. (Radiodrammi)

Forni, Marco, Nia y n pue’ de plu - Nulla e poco più, San Martin de Tor: Istitut «Micurà de Rü», 1993. (Poesie-gardenese-italiano)

Gruber, Tresele, $N$ pensier al dì, Bolzano, s.a. (Aforismi)

Moroder, Adele de Lenèrt, Stories, Urtijei: U.L.G., 1990. (Narrativa)

Mussner, Gudrun, Cuntia de na gabiana y de n giat che ti à nsenià da julé, San Martin de Tor: Istitut «Micurà de Rü», 2001. (Prosa-traduzione dallo spagnolo)

Piazza, Frida, L Mont di Vichings. Urtijei: Union di Ladins de Gherdeina. (Per bambini) Piazza, Frida, L Nost, In: Belardi, Walter: Narrativa Gardenese: Roma - Ortisei, 1988. (Romanzo)

Piazza, Frida, La cater sajons, Urtijei: Union di Ladins de Gherdeina. (Saggistica)

Piazza, Frida, La marueies de nosc albierch, Urtijei: Union di Ladins de Gherdeina, 1988. (Saggistica)

Piazza, Frida, La udera da la Duleda, Bulsan: U.L.G., 1991. (Poesie)

Piazza, Frida, Menizles. Tradujedes de poesies curtes o pertes de poesies. s.a. (1999).

Piazza, Frida, Pitla ustoria dla eves, Bulsan: Presel, 1977. (Saggistica)

Piazza, Frida, Stories de Anton Cechov. Urtijei 1968. (Prosa-traduzione)

Piazza, Frida, Taialonges y taiacurtes per majri y mendri, Urtijei: Typak, 1968. (Poesie per bambini)

Piazza Frida, Ustories d'Avent per mendri y majri, Bulsan: st. Presel, nuember 1990. (Prosa)

Pitschieler, Babi da Nudrëi, Sa Bula zacan, Urtijei: U.L.G., 1997. (Prosa)

Runggaldier Moroder, Ingrid, N viac tres la Dolomites, Urtijei: U.L.G., 1992. (Prosatraduzione dall' inglese)

Runggaldier Moroder, Ingrid, Urtijei y la Dolomites ti prim ani de nosc secul, Urtijei: U.L.G., 1995. (Prosa-traduzione dall' inglese)

Senoner, Elisabeth, Pitla stories dla bonanuet, Urtijei: U.L.G. 1995. (Racconti per bambini)

Senoner Mair, Erica, L pitl dragon conta, San Martin de Tor: Ist. «Micurà de Rü», 1993. (Racconti per bambini)

Senoner Mair, Erica, Max y Moritz. Set trifes de doi mutons. Urtijei: U.L.G., 1984. (Racconti per bambini)

Senoner Mair, Erica, Stories de Tieres, San Martin de Tor: Ist. «Micurà de Rü», 1987. (Racconti per bambini)

Tosi, Max, Ciofes da Mont, Urtijei: Union di Ladins de Gherdëina, 1975. (Poesie e prosa)

Verra, Roland, L cudejel di dis y dla sajons, Bulsan: U.L.G., 1989. (Poesie)

Verra, Roland, Dërc y storc, Bulsan: U.L.G., 1990. (Poesie)

Verra, Roland, Sun lim de plata, Urtijei: U.L.G., 1993. (Poesie)

Verra, Roland, Te me ancontes sën. Du triffst mich jetzt. Mi incontri adesso, Urtijei: U.L.G., 1997. (Poesie-trilingue) 


\section{Altri autori:}

Kostner, Josef (Poesie); Comploi, Hubert; Demetz, Georg; Demetz, Giudo (Teatro); Kasslatter, Hagen («Brunsin»); Mussner, Resi (Poesie); Runggaldier, Elsa (Traduzioni); Senoner, Toni, Helmuth, Insam (Prosa in lingua tedesca).

\section{Badia y Mareo / Val Badia con Marebbe:}

Baldissera, Pio; Irsara, Lois, Pëna y Pinel. Impresciuns söla natöra y söla jënt, Rezia n. 18, Bulsan, s.a. (Poesie)

Dapoz, Felix, In Banun. San Martin de Tor: Ist. «Micurà de Rü», 1982. (Poesie)

Flöss, Erna, Le descendent. De Cla Biert, San Martin de Tor: Ist. «Micurà de Rü», 1. pert 1995, 2. pert 2002. (Prosa - traduzione dal vallader)

Gasser, Tone, Danter dlijia y defora. Rimes, U.L.VB., 1994. (Poesia)

Morlang, Angelo, Fanes da zacan, San Martin de Tor: Ist. «Micurà de Rü», 1978. (Prosa in versi)

Morlang, Angelo, Sonn de ciampanes, San Martin de Tor: Ist. «Micurà de Rü», 1988. (Romanzo)

Morlang, Angelo, Vijins, San Martin de Tor: Ist. «Micurà de Rü», 1992. (Romanzo) Palfrader, Ingrid, La bradlada, Edizione propria, 2001. (Poesie)

Pescollderungg, Giuvani, Chilò orun restè, Bulsan: U.M.L., 1991. (Poesie)

Ploner, Iaco, Sön tru cun incherschimun. Ert por i ladins, 2000.

Rigo, Iaco, Da doman le ciarü, Eppan: edizione propria, 1991. (Romanzo)

Rigo, Iaco, La fata, Volterra-Prag-Sanct Petersburg-Pliscia: edizione propria, 1993. (Romanzo)

Rigo, Iaco, La maschera, Pliscia: edizione propria, 2000. (Romanzo)

Rigo, Iaco, Les vites de Elena R, Pliscia: edizione propria, 1994. (Romanzo)

Rigo, Iaco, Magheies, U.L.V.B., 1995. (Poesie)

Rigo, Iaco, Momonc, Edizione propria, 1990. (Poesie)

Taibon, Mateo, Cuintet Antich. Cin dialoghs y cater danterlüdi. (= Biblioteca di ricerche linguistiche e filologiche, 39), Roma: Il Calamo, 1996. (Dramma)

Trebo, Angelo, Poesies - Poet ladin (1862-1888), a cura di H. Dorsch-Craffonara, U.M.L., 1988. (Poesie)

Zardini, Paolo, Albeggia il tramonto, Roma: Vincenzo lo Faro ed., 1984. (Poesie)

Zardini, Paolo, La tornadöra, U.L.V.B., 1994. (Poesie)

Zardini, Paolo, Deboriada. Ert por i Ladins, 1998. (Poesie)

\section{Altri autori:}

Palfrader, Tresele (Poesie), Dapunt, Roberta (Poesie in lingua italiana); Leo, Irsara (Canzoni); Pedevilla, Josef; Anvidalfarei, Monika (Poesie in lingua tedesca); Trebo, Alessandro (Musicista-poesie); Christian Ferdigg (Poesie in lingua tedesca). 


\section{Fascia / Val di Fassa:}

Del Garber, Francesco, Rime Fashane, Vich / Vigo di Fassa: Ist. Cult. Lad. «majon di fascegn", 1987. (Poesie)

Dell'Antonio, Stefen Monech, Mudazion. Storie ladine, Moena: Grop Ladin da Moena, 1997. (Prosa)

Dell'Antonio, Valentino, Do l troi de la speranza, Grop Ladin da Moena.

Ghetta, Frumenzio P., Mizàcole de steile. Rimes fashanes, Vich / Vigo di Fassa: U.L.F., 1987. (Poesie)

Sorapera, Claus, Olà este pa?, Vich / Vigo di Fassa: U.L.F., 1994. (Poesie)

Sorapera, Claus, Poesies, Vich / Vigo di Fassa: U.L.F., 1996. (Poesie)

Soraperra, Simone, Usanzes e lugeres da zacan, Vich / Vigo di Fassa: Ist. Cult. Lad. «majon di fascegn».

Zanoner Piccoljori, Veronika, Leam ke rekonjonc, Moena: Grop Ladin da Moena, 1987. (Poesie)

\section{Altri autori:}

Angius, Luca; Sommariva, Simone; Jellici, Luciano; Iori, Vigile; Zanoner, Riccardo.

\section{Livinallongo / Fodom:}

Masarei, Sergio (Poesie; Usc di Ladins); Deltedesco, Marco.

\section{Cortina d'Ampezzo / Anpezo:}

Da Col, Gemo, Os de la val, 1985. (Poesie di Deltedesco Franco; Del Fabbro Ossi Iside; Dibona Marco; Majoni Ernesto)

Antologie e riviste:

Belardi, Walter, Antologia della lirica ladina dolomitica. Roma, 1985.

Belardi, Walter, Narrativa Gardenese. (= Biblioteca di ricerche linguistiche e filologiche 21), Roma Urtijei: Univ. «La Sapienza» - Union di Ladins de Gherdëina, 1988.

Belardi, Walter, Poeti ladini contemporanei. (= Biblioteca di ricerche linguistiche e filologiche, 16). Roma: «La Sapienza», 1985.

Bernardi, Rut; Locher Elmar; Mall Sepp; SAV, Leteratura, Literatur, Letteratura. Texte aus Südtirol. Bozen: ed. Sturzflüge, 1999.

Brunsin (L'). Plata de atualità y cultura (una volta al mese). (1982-1993).

EGHES. Movimënc tla leteratura ladina d'al dédancö, a cura de Roland Verra, Lia ScritourEs Scurlins. 1998. (Trilingue)

Poejies ora dl Brunsin 1982-1988, Urtijei: edizion «L Brunsin», 1988.

Poesies de ra nostres, a cura di Dibona Bonèla, Emanuela; Majoni Coleto, Ernesto,

1-5. ULdA, 1987-1991. (Più volumi) (Poesie ampezzane)

«Sckat». Rivista de ert y cultura. Numer unich, Urtijei: Lia Mostra d'Ert / Circolo, 1982. 
«Sckat». Rivista de ert y cultura. Grödner Jugend. Numero speciale. Urtijei: Lia Mostra d'Ert, 1984.

«Sturzflüge». Die Kulturzeitschrift. Holzwort Ladinien, Nr. 40/41, Jg. 12. Bozen, Okt.-Nov. 1994. (Con contributi in lingua ladina)

«Tras». - forum culturel. (Giornale culturale - letterario trilingue), Urtijei: U.G.L.D., $1994 \mathrm{f}$.

Rubatscher, Maria Veronika, Altgrödner Geschichten, Bozen: Manfrini V., 1981. (In lingua tedesca)

Rubatscher, Maria Veronika, Vedla Stories de Gherdeina. Tradotte in gardenese da Runggaldier Elsa, Urtijei: Union di Ladins, 1981.

Wolff, Karl Felix, L ciavalier cun la mirandules y d'autra liejendes. Tradotte da Runggaldier Elsa, Urtijei: ULG, 1988.

Wolff, Karl Felix, L reiam de Fanes y d'autra liejendes. Tradotte da Runggaldier Elsa, Urtijei: ULG, 1985.

Wolff, Karl Felix, Liejendes dla Dolomites. Tradotte da Runggaldier Elsa, Urtijei: ULG, s.a.

Nel mondo culturale ladino l'interesse per la letteratura è presente da molto tempo ma non c'è mai stata una struttura organizzata in modo efficace. Venivano pubblicate saltuariamente poesie in calendari, o su riviste, nello "Sckat» oppure nel «Brunsin» (vedi sopra), ecc. L'unico gruppo costituitosi e riconosciutosi come gruppo di scrittori è stato per molto tempo quello degli "Agacins» (cornacchie), un gruppo di giornalisti ancora presenti anche se poco attivi.

Dal 1993 ci sono $i$ "ScurlinEs» ${ }^{44}$. Scur = scuro e linëus = chiaro. Gli scurochiari oppure i chiaro-scuri. Un insieme di persone che all'inizio si riunivano solo per parlare di letteratura e confrontarsi sullo scrivere. Ora, per motivi formali, sono un gruppo organizzato in associazione ed hanno un loro statuto. $\mathrm{Ne}$ fanno parte persone provenienti da 3 vallate ladine (Gardena, Badia e Fassa).

Per molti di essi il gruppo è stata la prima occasione di confronto e di chiarificazione sulla propria coscienza letteraria. Oltre a produrre di più, fondarono anche una rivista in cui pubblicare i loro testi.

\section{La rivista letteraria TRAS:}

Questo forum culturale multilingue viene pubblicato dal 1994 una volta all'anno scegliendo di volta in volta una tematica specifica:

1. Mudamënc (Cambiamenti);

2. Sté (Stare / essere);

3. Autramënter (Diverso);

4. Dal sentiment al segn (Dal sentimento al segno)

5. Globalisazion (Globalizzazione)

6. Tabù (Tabù)

44. Rut Bernardi, Roberta Dapunt, Christina De Grandi, Stefen Dell'Antonio, Marco Forni (presidente dei Scurlins e redattore principale di Tras), Vigile Iori, Josef Pedevilla, Erica Senoner, Claus Sorapera, Mateo Taibon, Alessandro Trebo, Daria Valentin, Roland Verra, Riccardo Zanoner. 
7. Tacà te $\mathrm{n}$ fil (Attaccato ad un filo)

8. Euro [...] pa? (Veramente Europa?)

9. Arcipel ladin (Arcipelago ladino)

Ma qual è il significato dell'avverbio TRAS? È la forma ladino -«brach», in uso in metà della val di Fassa, con il significato di «attraverso; del tutto; per; per mezzo di; durante, sempre, ecc.»

$\begin{array}{ll}\text { son mol tres } 45 & \text { sono bagnato fradicio } \\ \text { furè tres ora } & \text { traforare } \\ \text { tres ite / tres ca / tres ju / tres su / tres via } & \text { dentro / in qua / in giù / in su / in via } \\ \text { rumè tres } & \text { cacciare } \\ \text { tres y tres } & \text { sempre, sempre di nuovo, attraverso del tutto } \\ \text { tresora } & \text { tutto il tempo } \\ \text { se n pië tres } & \text { scappare, andarsene }\end{array}$

Nella rivista «TRAS» non si trovano solamente testi creativi, ma anche saggi su problematiche riguardanti la tematica proposta. Vi sono inoltre anche recensioni di pubblicazioni nuove ed articoli vari sull'arte. Inoltre ogni numero della rivista viene curato graficamente da un / a artista locale.

Le lingue utilizzate sono: 4 idiomi ladini delle Dolomiti (gardenese, badiotto / marebbano, fassano, fodom) e il ladin dolomitan, la lingua ladina scritta unificata. $\mathrm{Ci}$ sono anche contributi in ladino e in romancio del Canton Grigioni, in friulano, tedesco, italiano ed una volta c'è stato un contributo anche in sloveno.

Credo di poter affermare che la rivista TRAS è un esempio di come i ladini siano in realtà gli unici a concretizzare il plurilinguismo tanto lodato in Alto Adige. Purtroppo nella maggior parte dei casi i ladini non vengono presi molto in considerazione. La ragione si potrebbe cercare nelle scarse competenze linguistiche dei sudtirolesi non ladini.

TRAS è la prima rivista culturale-letteraria dell' Alto Adige che include tutti i sudtirolesi e che non discrimina a seconda della lingua. La rivista va TRAS-ATTRAVERSO tutte le frontiere e lingue e meriterebbe più attenzione da parte del resto dell'Alto Adige e del Trentino.

\section{L'antologia EGHES:}

Eghes = Acque, movimenti nella letteratura ladina contemporanea, a cura di Roland Verra, edita dalla «Lia ScritourEs ScurlinEs» nel 1998 è un'antologia trilingue. Eghes non è un'antologia classica, ma una selezione di persone di madrelingua ladina che scrivono. Sono stati scelti 4 autori / autrici di ogni vallata (Gardena, Badia, Fassa). Di ogni «scrittore» si può leggere una biografia, una critica delle sue opere letterarie e cinque testi letterari. Il curatore di Eghes, Roland Verra, fa anche un quadro della situazione letteraria della Ladinia. Eghes è un testo che potrebbe essere fruibile anche nelle scuole. 


\subsection{Come e cosa scrivono?}

Credo di poter affermare che questi «scriventi» scrivono diversamente dai cosiddetti «autori ladini classici» che troppe volte vengono strumentalizzati come scrittori alibi. ${ }^{46}$ Gli scrittori d'oggi sono diversi, hanno usufruito di un'altra istruzione scolastica, vivono diversamente, hanno mestieri diversi, vedono il mondo in maniera diversa, pensano e cioè scrivono diversamente.

Ma la cosa che li differenzia di più dai cosiddetti «autori classici» è la motivazione della scrittura, e non sta a me giudicare se sia migliore o peggiore.

Per quali ragioni scrivevano un tempo?

- Per conservare parole ed espressioni idiomatiche antiche

- Per diffondere una dottrina

- Per raccontare avvenimenti storici

- Per criticare e offendere

Se un tempo si scriveva per ragioni conservative rispetto alla lingua e alla cultura, oggi si scrive il più delle volte per se stessi, per egoismo oppure semplicemente per il piacere di scrivere. Si potrebbe anche dire che la tendenza all'individualismo nella nostra società si rispecchia anche nello scrivere. Raramente si riscontrano oggi testi letterari volti meramente alla conservazione della lingua oppure elenchi di parole da salvare, quali gli arnesi contadini.

Anche le diatribe per singole espressioni idiomatiche, per grafemi oppure accenti ortografici appartengono per fortuna al passato. L'importanza della coscienza letteraria è cresciuta.

Il mondo di questi, diciamo, giovani scrittori, ha in ogni senso, già da tempo, varcato i confini. Non solamente nei contenuti, ma anche nella lingua: alcuni scrivono in lingua italiana o tedesca. La lingua principale è però rimasta il ladino e ciò lo si può interpretare alla luce di varie motivazioni. Si scrive in ladino per rintracciare le radici perdute, rendendosi conto a volte che, con testi esotici, oggi si può attirare più facilmente l'attenzione. Scrivere in ladino è però sicuramente una scelta consapevole.

L'elaborazione di tematiche storiche è sempre stata caratteristicha delle letterature di lingue meno diffuse, così anche per quella ladina. È importante notare che oggigiorno le caratteristiche più significative degli «scriventi» sono sicuramente la tendenza verso il minimalismo e le tematiche della vita di tutti i giorni. Nell'attuale letteratura saggistica ladina mancano però quasi totalmente le riflessioni sulla letteratura in senso stretto. Nelle cosiddette "piccole comunità linguistiche» la letteratura viene ancora spesso trattata da matrigna.

46. Solo per una presenza d'obbligo del ladino. 


\section{Che cosa si può dire in termini scientifici sulla produzione letteraria ladina?}

Iso Camartin, filosofo e scienziato di letteratura grigionese afferma lucidamente: «Man darf sich die Entwicklung von Kleinkulturen nicht nach dem Muster der Großkulturen denken, wenn man seine Enttäuschungen nicht bereits vorprogrammieren will.» ${ }^{47}$

Da un lato sottolinea questa grande libertà di non doversi misurare con le norme di grandi letterature, e dall'altro lato dice: «Ein jiddischer Schriftsteller hat einmal davon gesprochen, dass seine Sprache die Literatur einwickle wie in eine Fettschicht, die schützt, aber auch hoffnungslos isoliert [... ${ }_{\gg}{ }^{48}$

Lo scrivere senza norme prestabilite può essere più facile e in alcuni casi anche più libero, perchè si possono produrre testi del tutto innovativi. D'altra parte può anche essere molto difficile scrivere senza tradizione e senza modelli letterari. Se nel passato questi modelli mancavano soprattutto alle scrittrici, nelle letterature di lingue meno diffuse mancano anche agli scrittori. Nelle letterature di lingue meno diffuse valgono per entrambi i sessi gli aspetti positivi come anche quelli negativi.

\subsection{Contraddizioni nella letteratura ladina}

La mancanza di astrazione e estetismo vs. purismo linguistico

Da un lato abbiamo la mancanza di astrazione, sia da parte degli «scriventi» come anche da parte dei lettori. Da ciò risulta inevitabilmente una mancanza di estetismo nei testi letterari sia a livello di contenuto che a livello ortografico.

L’obbligo di dover «scrivere come si parla» è come la spada di Damocle che pende sopra la testa degli «scriventi» in una lingua meno diffusa. «[...] nur eines scheint zu fehlen: die Transformation vom Leben zur Kunst», ${ }^{49}$ osservano Lucia Walther e Cla Riatsch, nel loro saggio sulla letteratura romancia nel Canton Grigioni del XX secolo. Il gioco di riconoscere nei testi letterari i luoghi e i personaggi di un ambiente così piccolo è un'oggetività che inganna, perchè non basta rispecchiare la realtà; bisogna anche trasformarla in creazione artistica.

Peter Bichsel scrive che all'autore «non importa solo del contenuto, ma della riflessione, del raccontare e della metodologia del raccontare.» ${ }^{50}$ «Das scheint

47. Iso CAmartin, Nichts als Worte. Ein Plädoyer für Kleinsprachen, Zürich: Ex Libris, 19872, p. 164. (Non si può pensare lo sviluppo di piccole culture come i modelli delle culture grandi, se non si vuole essere delusi già in anticipo.)

48. Ibidem, p. 165. (Uno scrittore ebreo un bel giorno ci raccontò che la sua lingua avvolge la letteratura come in uno strato di unto per proteggerla, ma nello stesso tempo la isola estremamente $[\ldots]$.

49. Cla Riatsch,; Lucia Walther, Literatur und Kleinsprache. Studien zur bündnerromanischen Literatur seit 1860. (=Romanica Raetica 11, 12), Chur: Societ. Retorumantscha, 1993, p. 784. ([...] solo una cosa sembra che manchi: la trasformazione dalla vita all'arte.)

50. Cfr. Peter BiChSEL, Der Leser. Das Erzählen, Frankfurter Poetik-Vorlesungen, Darmstadt: Luchterhand V., 1982, p. 78. 
mir eine wesentliche Aufgabe der Literatur zu sein: dafür zu sorgen, daß wir nicht nur die Geschichte, sondern auch die Geschichten erkennen können. Es geht nicht einfach nur um das Erkennen von Realität, es geht darum, die Realitäten in eine humane Tradition einzubringen, in die Tradition des Erzählens. [...] Die Literatur hat die Aufgabe und den Sinn, die Tradition des Erzählens fortzusetzen, weil wir unser Leben nur erzählend bestehen können.» ${ }^{51}$

D'altra parte nelle letterature di lingue meno diffuse c'è una certa tendenza al purismo che si manifesta in una messa in scena di se stessi e in un conservativismo provocato dall'aggrapparsi alla lingua e dal rispetto della tradizione. Un purismo linguistico che, per amore della lingua materna rischia di frenare un possibile sviluppo naturale e scientifico della stessa.

Questa contraddizione nasce dalla paura dell'estinzione della lingua, sorta già nel 1900, quando si sviluppò la coscienza che il ladino fosse una lingua e i ladini un popolo a se stante.

\subsection{Uno sguardo alla lirica ladina}

Lo scrittore Reto Caratsch di lingua ladina-puter dell'Engiadina Alta «führt das Überwiegen der "kleinen Form" in der bündnerromanischen Literatur auf die zu hohen Druckkosten zurück, die bei längeren Texten entstünden, weshalb die Autoren vornehmlich dazu angehalten würden, Gedichte zu schreiben.» ${ }^{52}$ Viene osservato che la forma artistica letteraria più alta e difficile è la poesia. Gottfried Benn, dice nel 1951 in «Probleme der Lyrik. Marburger Rede»: «Mittelmäßige Poesie ist nicht erlaubt und unerträglich.» (La poesia mediocre non è permessa ed è insopportabile).

R. M. Rilke scrive in una lettera del 17.02. 1903 ad un giovane scrittore: «Schreiben sie nicht Liebesgedichte; weichen sie zuerst denjenigen Formen aus, die zu geläufig und gewöhnlich sind: sie sind die schwersten, denn es gehört eine große, ausgereifte Kraft dazu, Eigenes zu geben, wo sich gute und zum Teil glänzende Überlieferungen in Menge einstellen.» 53

51. Peter BICHSEL, Der Erzähler. Das Erzählen. Frankfurter Poetik-Vorlesungen, Frankfurt: Luchterhand V., $1989^{5}$, p. 83. (Questo mi pare uno dei compiti fondamentali della letteratura: di occuparsi che non si intraveda solo la storia, ma che si riconoscano anche le storie. Importante non è solamente il riconoscere la realtà, ma introdurre la realtà in una tradizione umana, nella tradizione del raccontare. [...] La letteratura ha il compito e lo scopo di continuare la tradizione del raccontare, perchè unicamente raccontando siamo in grado di superare la nostra vita.)

52. Cla RiatsCH; Lucia Walther, Literatur und Kleinsprache. Studien zur bündnerromanischen Literatur seit 1860. (=Romanica Raetica 11, 12), Chur: Societá Retorumantscha, 1993, p. 213. (Reto Caratsch dice nel 1983 che «il prevalere della "forma piccola" nella letteratura romancia nei Grigioni è da cercare nelle spese troppo alte della stampa che comporterebbero testi più lunghi. Per questa ragione viene consigliato agli autori di scrivere poesie».)

53. Rainer Maria RILKE, Briefe an einen jungen Dichter. Frankfurt e Leipzig: Insel V., $1992^{40}$, p. 8. (Non scrivete poesie d'amore; evitate all'inizio quelle forme che sono troppo diffuse e semplici: sono le più difficili, perchè ci vuole una forza enorme e una grande maturità per scrivere cose proprie in una forma dove c'è già una grande e splendida tradizione.) 
È interessante poter leggere in Benn che la poesia è la forma d'arte letteraria più difficile e in Rilke che le tematiche semplici e quotidiane sono le più difficili per una trasformazione letteraria. Non troviamo giustamente nella letteratura ladina spesso questi due elementi, poesie e tematiche semplici?

\section{E le muse?}

Ci permettiamo di affermare come dice Gottfried Benn che la gente pensa che una poesia nasca in questo modo: un bel giovane oppure una bella ragazza con mal d'amore seduta su una panchina, osservando un bel tramonto, sente il bisogno di esprimere i propri sentimenti e scrive una poesia. Ciò è assurdo, perchè una poesia in ogni lingua deve venir scritta ed elaborata e non viene ispirata dall'alto. Scrivere poesie oggigiorno non è come scrivere la Bibbia, che, probabilmente, è nata dall' ispirazione di Dio! Moltissimi scrittori noti lavoravano (e lavorano) come funzionari nel loro ufficio, tutto il santo giorno! E se una musa che ispira poesie dovesse veramente esistere mi chiedo che cosa stia aspettando per ispirare finalmente qualche testo o poesia geniale in ladino!

Concludendo vorrei riproporre per la letteratura ladina al posto di «piccola letteratura» o "letteratura di minoranza» il termine «letteratura di una lingua meno diffusa», perchè con $\mathrm{i}$ termini «piccola» $\mathrm{o}$ «minore» oggi certe poesie o testi letterari in ladino, non hanno più niente in comune.

E a dire la verità, non ho mai pensato che la nostra letteratura ladina sia «di confine». Per me è diventata quella più vicina, quella al centro della mia vita e professione. La parola "confine» è troppo artificiale, arbitraria e politica, e ha poco a che fare con i sentimenti di appartenenza delle persone. I confini vengono fatti dalla politica e non corrispondono alle appartenenze linguistiche e culturali. Che la letteratura latina sia però una letteratura sull'orlo o sul bordo, nel senso di poca diffusione e sviluppo con inerzia, era sempre evidente.

\section{Esempio letterario}

$\begin{array}{llllll}\text { la } & \text { ie } & \text { pa } & \text { da } & \text { rì } & \\ \text { é } & \text { pa } & \text { mé } & \text { da } & \text { dì } & \\ \text { la } & \text { ie } & \text { da } & \text { tò } & \text { y } & \text { jì } \\ \text { n } & \text { ne } & \text { sà } & \text { pa } & \text { co } & \\ \text { fé } & \text { a } & \text { dì } & \text { mo } & \text { a } & \text { vo } \\ \text { te } & \text { n } & \text { di } & \text { o } & \text { no } & \\ \text { l } & \text { cë } & \text { ne } & \text { va } & \text { pa } & \text { mé } \\ \text { a } & \text { jì } & \text { do } & \text { si } & \text { pe } & \\ \text { la } & \text { ne } & \text { ie } & \text { pa } & \text { da } & \text { rì } \\ \text { co } & \text { ne } & \text { sà } & \text { no } & \text { èi } & \\ \text { y } & \text { no } & \text { si } & \text { fi } & \text { da } & \text { dì } \\ \text { la } & \text { ie } & \text { mé } & \text { da } & \text { tò } & \\ \text { y } & \text { de } & \text { ne } & \text { dì } & \text { no: } & \text { oh } \\ \text { da } & \text { dì } & \text { do } & & & \end{array}$




\section{Traduzione:}

Fa proprio ridere

voglio solo dire
pigliala e vai
non si sa come
fare a dire ancora a voi
in un giorno o no
la testa non funziona solo
a seguire il suo piede
non fa proprio ridere
come non sanno dire né loro
e neanche il loro figlio
va solo presa
e che non si venga dire: oh
da ripetere

\section{Bibliografia scelta}

ALEWYN, Richard, Probleme und Gestalten. Essays, Frankfurt: Suhrkamp, $1988^{6}$.

BELARDI, Walter, Max Tosi poeta ladino, Firenze: Ist. di studi per l'Alto Adige, 1985.

BERNARDI, Rut, Curs de Gherdëina. Trëdesc lezions per mparé la rujeneda de Gherdëina. Dreizehn Lektionen zur Erlernung der grödnerischen Sprache, Zürich, Innsbruck: St. Ulrich, Ist. Cult. Lad. «Micurà de Rü», 1999. (Trëdesc lezions per mparé la rujeneda de Gherdëina. Tredici lezioni per imparare la lingua gardenese, Zurigo, Innsbruck: Ortisei, Ist. Cult. Lad. «Micurà de Rü», 2002).

Bernardi, Rut; DeCurtins, Alexi; EichenhOFER, Wolfgang; SaluZ, Ursina; VöGELI Moritz, Handwörterbuch des Rätoromanischen. Erarbeitet auf Initiative von Hans Stricker. [Bd. I: A-M; Bd. II: $N-Z$; Bd. III: Indizes], Zürich: Società Retorumantscha und Verein für Bündner Kulturforschung - Offizin, 1 (1994) 1-510; 2 (1994) 521-1022; 3 (1994) 1031-1567. (Wortschatz aller 5 rätoromanischen Schriftsprachen Graubündens, einschliesslich Rumantsch Grischun, mit Angaben zur Verbreitung und Herkunft). Rc.: Mondo Ladino 18 (1994) 302-304 [Plangg, Guntram A.]; Vox Rom. 54 (1995) 276-279 [Liver, Ricarda]; Zs. Rom. Philol. 112 (1996) 839-840 [Pfister, Max].

Bernardi, Rut, «Frida Piazza. Die ladinische Schriftstellerin». In: Sturzflüge. Die kulturzeitschrift. Nr. 40/41 - Okt./Nov. 1994, p. 49-50.

BERNARDI, Rut, «Gälisch: Die keltische Sprache der Gälen in Schottland». In: Mondo Ladino. Boletin de l'Istitut Cultural Ladin. Ann XVII (1993) n. 3-4, p. 89-109.

BERNARDI, Rut, «Gedanken - Collage: Ladinische Literatur heute». In: Kulturelemente, Nr. 7, 1998, p. 5-6.

BERNARDI, Rut, «L ladin dla Dolomites: Planificazion, elaborazion y normalisazion tres standardisazion». In: Mondo Ladino. Bolatin de l'Istitut Cultural Ladin. Ann XVII (1993) n. 3-4, p. 37-65.

BERNARDI, Rut, «Ladinische Literatur heute, Gibt es sie wirklich?» In: Literathek. Zum Lesen / Südtiroler Bibliotheks-Info. Sondernummer zur 47. Frankfurter Buchmesse 95, p. 20-22. 
BEzZEL, Chris, Wittgenstein zur Einführung. Hamburg: Junius V., $1989^{2}$.

Bezzola, Reto R., Litteratura dals Rumauntschs e Ladins, Cuira: Lia Rumauntscha, 1979.

BICHSEL, Peter, Der Erzähler. Das Erzählen. Frankfurter Poetik-Vorlesungen, Frankfurt: Luchterhand V., $1989^{5}$.

BiCHSEL, Peter, Der Leser. Das Erzählen. Frankfurter Poetik-Vorlesungen, Darmstadt: Luchterhand V., 1982, p. 78.

BICHSEL, Peter, "Kindergeschichten», Darmstadt und Neuwied: Luchterhand V., 1976, 6. ed. («Stories per mendres y menders.»)

CAMARTIN, Iso, Nichts als Worte. Ein Plädoyer für Kleinsprachen, Zürich: Ex Libris, $1987^{2}$.

ChiocchetTi, Fabio, "Appunti per una storia della letteratura ladina dolomitica». In: Mondo Ladino XXIV (2000), Vigo di Fassa: Ist. Cult. Lad. «majon di fascegn».

Chiocchetti, Fabio. † Hugo de Rossi (1875-1936). "Ko ke la é stada ke son ruà sul bal dei Dolomitenladiner». In: Mondo Ladino VI, n. 1-2 (1982), Vigo di Fassa: Ist. Cult. Lad. «majon di fascegn», p. 121-191.

ChIocChetTI, Nadia (redatoura prinzipala); BERnARDI, Rut; CHIOCCHETTI, Fabio; Gsell, Otto; Stuflesser, Mathias; Valentin, Daria; VidesotT, Paul, Gramatica dl Ladin Standard, Vich, San Martin de Tor: Bulsan, Spell, 2001.

CiOran, E. M., Gevierteilt, Frankfurt: Suhrkamp Tbv., 1991.

Cioran, E. M., Vom Nachteil, geboren zu sein. Frankfurt: Suhrkamp Tbv., 1979.

Daltone, Alvijo (mareo); ObleTter, Amalia (gherdeina), Ciüria chécena; Cazina cuecena, San Martin de Tor: Ist. Ladin «Micurà de Rü», 1981.

DECURTINS, Alexi, «Entgins patratgs davart la Crestomazia e siu editur». In: Viarva romontscha II. (=Romanica Raetica 9), Cuira, Societad Retorumantscha, 1993.

DeCuRTINs, Caspar, Rätoromanische Chrestomathie. 13 Vol., Erlangen, 1896-1919.

DERUNGS, Ursicin G. G. «Entusiassem per sligiaziuns magicas». In: Litteratura. Novas litteraras. Rumantsch grischun. 13. USR: Cuira, 1990, p. 23-24.

DILF: Dizionario italiano - ladino fassano, Vich / Vigo di Fassa: Ist. Cult. Lad. - Spell, 1999.

GERLIN, Toni; Maly, Anton, Le Maradët. traduziun: Willeit Iustina y Ulrich. (= Sêria «Teater», nr. 3), San Martin de Tor: Ist. Ladin «Micurà de Rü», 1984.

GIGER, Felix, «Editorial». In: Litteratura. Novas litteraras. Rumantsch grischun. 13. USR, Cuira, 1990, p. 9-10.

GIGER, Felix, "Rumantsch grischun sco lingua litterara». In: Litteratura. Novas litteraras. Rumnatsch grischun. 13. USR, Cuira, 1990, p. 16-20.

KINDL, Ulrike, "Kritische Lektüre der Dolomitensagen von Karl Felix Wolff. Bd.1: Einzelsagen». San Martin de Tor: Istitut Culturel Ladin «Micurà de Rü», 1983.

KNELLWOLF, Ulrich, "Tod in Sils Maria. 13 üble Geschichten», Zürich: Arche V., $1994^{2}$, («Mort tles Dolomites. 13 stories entortes.»)

KUHN, Heinrich, «Rätoromanisches bei Oswald von Wolkenstein». In: Ladinia 3, San Martin de Tor: Istitut Ladin «Micurà de Rü», 1979.

Litteratura. Novas litteraras, 13: «Rumnatsch grischun». USR, Cuira, 1990.

LÜDTKE, Jens, «OvW und die romanischen Sprachen». In: Logos semantikos. (Festschrift Coseriu). A cura di Horst Geckeler u. a, Berlin / New York: 1981, Bd. 1, p. 303-312.

PiCHLER, Anita; VAllazZA, Markus, Die Frauen aus Fanis, Innsbruck: Haymon V., 1992.

PiZZININI, Antone, Parores ladines. Vokabulare badiot - tudësk. Ergänzt und überarbeitet von Guntram Plangg, Innsbruck: (= Romanica Aenipontana), 1966.

Ploner, Iaco, A Fridl - Incarnaziun. A Federico - Incarnazione, S. Giorgio di Nogaro: ed. la bassa - poesia / 3, 1991, p. 28-31. 
RiatsCh, Cla; Walther, Lucia, Literatur und Kleinsprache. Studien zur bündnerromanischen Literatur seit 1860. (=Romanica Raetica 11, 12), Chur: Società Retorumantscha, 1993.

RiLKE, Rainer Maria, Briefe an einen jungen Dichter, Frankfurt und Leipzig: Insel V., $1992^{40}$.

SCHANIEL, Annalis, «Experientschas cun la translaziun litterara.» In: Litteratura. Novas litteraras. Rumnatsch grischun. 13. USR, Cuira, 1990, p. 36-37.

SCHATZ, Josef, «Die deutsche Sprache in Südtirol». In: Südtirol. Hg. K. v. Grabmayr, 1919.

SCHATZ, Josef, Sprache und Wortschatz der Gedichte Oswalds von Wolkenstein. (=Denkschriften, 69. Band, 2. Abhandlung), Wien und Leipzig, 1930.

SCHMID, Heinrich, Richtlinien für die Gestaltung einer gesamtbündnerromanischen Schriftsprache Rumantsch Grischun, Cuira: Lia Rumantscha, 1982.

SCHMID, Heinrich, Wegleitung für den Aufbau einer gemeinsamen Schriftsprache der Dolomitenladiner, San Martin de Tor: Ist. Cult. Lad. «Micurà de Rü»; Vich, Ist. Cult. Lad. "Majon di Fascegn», 1998.

SCHOPENHAUeR, Arthur, Die Welt als Wille und Vorstellung. Bd. I, Bd. II, Zürich: Haffmans V., 1988.

SPESCHA, Flurin. Fieu e flomma, Cuira: Octopus, 1993.

WACHINGER, Burghart (Hg.). Oswald von Wolkenstein. Lieder. Mittelhochdeutsch / Neuhochdeutsch, Stuttgart: Reclam Jun., 1980². 